\title{
European ENVISION Project for Improving Timing Resolution in PET Systems
}

\author{
J.F. OLIVER* on behalf of ENVISION members \\ Instituto de Física Corpuscular, IFIC (CSIC-UV), c/ Catedrático José Beltrán 2, E-46980 Paterna, Spain
}

\begin{abstract}
Hadrontherapy uses charged particles to destroy tumors. For improving the quality assurance tools, specially range verification, the European Commission funded the project ENVISION. This paper summarizes the activities carried out within the work package two devoted to improve the visualization of the spatial distribution of positron emitters that are produced during patient irradiation. Allowing the beam passage implies a reduction in the angular coverage which degrades the range assessment. The aim of the work package two was to investigate the potential use of time-of-flight information for compensating this degradation. The studies can be classified into hardware and software developments. For the former, several ASICs and DAQs have been investigated. Also, the potential application of resistive plate chambers has been assessed. Four dual-head demonstrators have been produced by the end of the project. The software developments are divided into simulations and image reconstruction. Several algorithms able to include the time-of-flight information have been developed. Direct reconstruction has been implemented and compared with conventional maximum-likelihood methods. The results show that direct time-of-flight for on-line reconstruction is possible although iterative algorithms can achieve further improvements. However, time-of-flight alone might not be sufficient due to the low emission rates.
\end{abstract}

DOI: $10.12693 /$ APhysPolA.127.1513

PACS: 87.57.nf, 87.53.-j, 87.56.Fc

\section{Introduction}

Hadrontherapy (HT) uses beams of charged particles to destroy tumor cells. In order to improve the quality assurance tools, the European Commission funded ENVISION [1] a 4-year project that aims at developing solutions for: real-time non invasive monitoring, quantitative imaging, precise determination of delivered dose, fast feedback for optimal treatment planning, real-time response to moving organs. Launched in February 2010, ENVISION was a collaboration of sixteen leading European research centers and industrial partners, coordinated by CERN. ENVISION was divided in seven work packages (WPs). Here, we summarize the activities that were carried out within the WP2: "Time-of-Flight inbeam PET'(ToF ib PET).

HT allows for a superior dose distribution than conventional X-ray techniques. However, this higher conformality poses the problem of range monitoring. The WP2 was conceived to address this issue by investigating one of the possible approaches to range verification. In particular, the final aim of the WP was to improve the visualization of the spatial distribution of $\mathrm{e}^{+}$emitters that are produced during patient irradiation in particle therapy. To this end, the application of the positron emission tomography (PET) technique was foreseen to be employed in-beam (ib), ie. without transferring the patient being irradiated to a different location for PET imaging. Not moving the patient has several advantages, such as, prevent alignment errors and decrease the negative impact of

*e-mail: oliver@ific.uv.es radio-isotope washout. However, the in-beam technique has a main drawback. Allowing the free passage of the beam implies a reduction in the angular coverage which, in turn, degrades the quality of the final image and, by extension, the quality of the range assessment.

The degradation due to limited angular coverage may be compensated by using ToF information. The research framed within this WP was aimed at assessing the potential of the ToF ib PET technique.

\section{Summary of the research activities}

The successful application of ToF ib PET relies on achieving sufficiently high time resolution. At the beginning, the goal was to achieve $200 \mathrm{ps}$ (FWHM) for the coincidence resolution time (CRT). In the search of high timing resolution towards ToF, four main lines have been followed: improve crystal-based PET systems, investigate alternative detectors, improve detector electronics and DAQs. These advances have been complemented with simultaneous developments of image reconstruction algorithms able to use the ToF information for reducing the truncation artifacts caused by limited angular coverage. The WP 2 developments can be grossly classified into hardware (instrumentation) and software developments.

\subsection{Instrumentation}

2.1.1. Development and comparison of technologies for sub-nanosecond ToF resolution

On-detector electronics developments have been mainly done in situ by the different groups. Basic studies of various ASIC technologies have been done. The ASICs were coupled to crystal-based and RPCbased PET demonstrators. In particular, the BASIC chip has been tested with SiPM for crystal-based detectors. The NINO chip has been tested with crystal-based 
detectors. With this chip a CRT $=220$ ps $(\mathrm{FWHM})$ has been achieved. Finally, the SPIROC chip has been tested on SiPM for crystal-based and RPC-based detectors. For the associated DAQs, three approaches have been explored and used. The BASIC DAQ System which was based on the BASIC chip, the VATA DAQ System which was adaptable to different detectors/ASICs and the $\mu \mathrm{TCA}$ DAQ System based on $\mu \mathrm{TCA}$ which was used for crystal-based as well as RPC-based systems.

In addition, an alternative detector approach has been investigated: the use of multigap resistive plate chambers (MRPC). The RPCs offered: promising timing properties compatible with the project goal, depth of information $(5 \mathrm{~mm})$, multi-hit resolution $(10 \mathrm{~mm})$ and the possibility of constructing large areas at low cost. Preliminary results suggest a $\sigma_{\mathrm{ToF}}=240 \mathrm{ps}$.

\subsubsection{Design, construction and testing of dual-head demonstrators}

Four crystal-based dual-head demonstrators have been built. Three of them are small ToF PET demonstrators with efforts put on ToF improvement while the fourth is an ib PET demonstrator for various beam types. The first of the three ToF PET demonstrators has been built at $\mathrm{CERN}^{\dagger}$ and has allowed comparing different technologies towards improved ToF. Several studies of timing performances of crystal matrices has been performed. The second demonstrator, 4DM-PET Module, is based on continuous LYSO crystal coupled to both sides with SiPM arrays. This prototype presents high spatial resolution in $x-y$ directions $(\approx 1.7 \mathrm{~mm} \mathrm{FWHM})$, DOI capabilities, insensitivity to magnetic fields and time resolution compatible with ToF requirements. The third crystal-based PET prototype consists of two planar heads each made of an $2 \times 4$ array of $3 \times 3 \times 10 \mathrm{~mm}^{3}$ LYSO crystal coupled to SiPMs. At the end of the project, the DAQ system (based on $\mu \mathrm{TCA}$ ) was being incorporated.

The main aim of the fourth demonstrator was the development of a true in-beam PET system able to separate true coincidences from nuclear background independently of the beam time structure for selecting events that are not correlated to beam spill. To this end two types of beams and time structures were considered: ${ }^{12} \mathrm{C}$ beams $(75 \mathrm{MeV} / \mathrm{u})$ and proton beams $(86 \mathrm{MeV})$.

\subsection{Software}

\subsubsection{Simulations of full ib ToF PET systems}

The complexity associated to the full chain (from patient irradiation to PET detection) was taken into account in combination with WP6. Figures-of-merit were agreed for the assessment of the systems. The simulations were based on Geant 4 and GATE. Three scenarios of increasing complexity were implemented. In the simplest one, a basic characterization of detector performance was done by using only back-to-back photons and

${ }^{\dagger}$ In combination with the Endoscopic ToFPET \&Ultrasound (ENDO ToF PET) project simple sources (points, lines, etc). In the intermediate scenario, single beams of proton and carbon ions were simulated together with water or PMMA phantom. Finally, the more realistic scenario took patient data from carbon-ion treatment plans.

To advance the performance of the future ib ToF PET system, several simulations with different configurations were done. In particular, RPC-based PET systems were simulated under various configurations. For crystalbased PET systems, the Philips GEMINI TF scanner was used as a reference. Several configurations were investigated varying the angular coverage, energy windows, diameter and time resolution. A basic performance assessment for the representative open configurations is shown in Table.

TABLE

Performance comparison. Basic performance assessment for the representative open configurations.

\begin{tabular}{c|c|c|c}
\hline \hline Scanner & $\begin{array}{c}\text { Sensitivity } \\
{[\mathrm{cps} / \mathrm{kBq}]}\end{array}$ & $\begin{array}{c}\text { Scatter } \\
\text { fraction }\end{array}$ & $\begin{array}{c}\text { Transaxial resol. } \\
{[\mathrm{mm}] \text { FWHM }}\end{array}$ \\
\hline crystal & 21.60 & $32.5 \%$ & 3.4 \\
RPC & 4.08 & $56.6 \%$ & 3.8
\end{tabular}

\subsubsection{Development of image reconstruction algorithms for ib ToF PET}

Image reconstruction faced two challenges: the data loss due to open geometries and the low emission rates. To deal with them, dedicated reconstruction algorithms were implemented for each system and fast methods for on-line reconstruction were developed.

In Fig. 1 the results obtained in the simulations for crystal-based and RPC-based systems are shown.

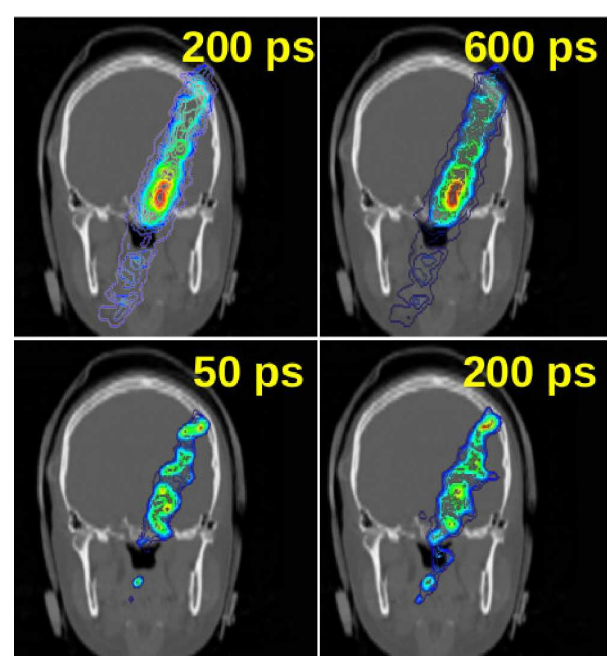

Fig. 1. Reconstructed annihilation maps obtained for crystal-based (top) and RPC-based (bottom). Time resolutions used are indicated.

The images are worse for the RPC case, even for high time resolutions of $50 \mathrm{ps}$. They are noisier than the crystal-based ones and, moreover, many abnormal 
amounts of annihilations are allocated beyond the distal edge.

Two approaches that used the ToF information were investigated: direct reconstruction (DR) and statistical iterative reconstruction (SIR). For the DR case, the voxel with the highest probability was taken to be the voxel where the annihilation took place while, for the SIR case, the full probability distribution is kept and embedded into conventional iterative techniques (MLEM, MAP).

The DR is a direct process (each detection is assigned a voxel independently of the rest of the detections) while the SIR, although more accurate, requires the simultaneous information of all detections, thus, forcing a slow post-acquisition reconstruction.

A comparison between images reconstructed using DR and SIR methods is shown in Fig. 2. Both methods provide acceptable images of the annihilation map. As expected, DR methods provide fast reconstructions at the cost of reducing the accuracy of the image.

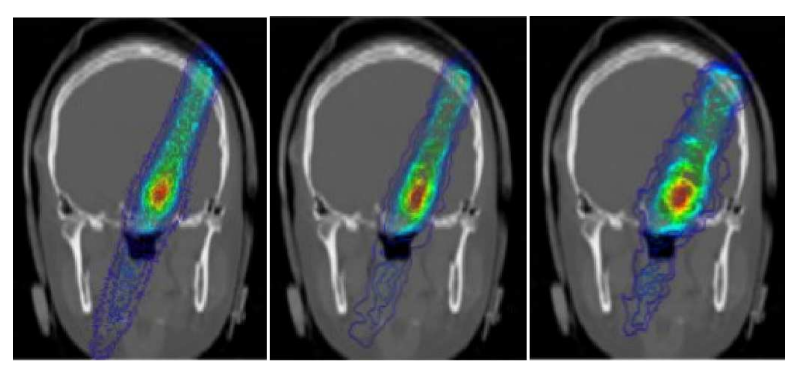

Fig. 2. The reference annihilation map (left), MLbased reconstruction (middle) and DR-based reconstruction (right).

\section{Summary and conclusion}

In the search of high timing resolution towards ToF values about 200 ps CRT have been achieved. Several detector concepts have been developed and promising alternatives for ibPET incorporating ToF have been assessed. If high enough, direct ToF for on-line reconstruction is possible. Still, iterative ToF reconstruction algorithms can achieve further improvements. However, ToF alone it might not be sufficient. Low emission rates remains as one of the main challenges for accurate reconstruction and range verification. For improving range verification, further improvements in image reconstruction are needed specifically adapted to in-beam conditions and, possibly, parametric reconstruction instead of image reconstruction.

\section{References}

[1] http://envision.web.cern.ch/ENVISION/. 